\title{
Intravitreal injection of crystalline cortisone as adjunctive treatment of proliferative vitreoretinopathy
}

\author{
Jost B Jonas, Jochen K Hayler, Songhomitra Panda-Jonas
}

\begin{abstract}
Aim-To report on clinical outcome and complications of intravitreal injection of crystalline cortisone in patients undergoing pars plana vitrectomy for treatment of proliferative vitreoretinopathy.

Methods-The study included all 16 patients who underwent pars plana vitrectomy for treatment of proliferative vitreoretinopathy, who received an intravitreal injection of 10-20 $\mathrm{mg}$ crystalline triamcinolone acetonide at the end of surgery, and who were operated on by the same surgeon. Most of the vehicle of the solution containing the cortisone crystals was removed before performing the injection. Mean follow up time was 1.64 (SD 2.15) months (median 1.23 months; range 0.20-9.20 months). The study group was compared with a control group which consisted of 144 patients undergoing pars plana vitrectomy for proliferative vitreoretinopathy performed by the same surgeon.
\end{abstract}

Results-In the study group compared with the control group, intraocular inflammation, as estimated clinically by slit lamp biomicroscopy, was lower, appearance of the fundus upon ophthalmoscopy in the first postoperative week was clearer, and postoperative pain in the first two postoperative days was reduced. Intraocular pressure measured at the end of the first postoperative week did not vary significantly between the groups. A pseudohypopyon consisting of cortisone crystals in the inferior anterior chamber angle was detected in one patient. Postoperative infectious endophthalmitis was not encountered.

Conclusions-This pilot study suggests that intravitreal injection of crystalline cortisone with most of the vehicle removed is not toxic to intraocular structures, reduces postoperative intraocular inflammation, and may be a potentially useful additional tool in the treatment of proliferative vitreoretinopathy.

(Br f Ophthalmol 2000;84:1064-1067)

Corticosteroids have been used in ophthalmology since the early 1950 s to suppress intraocular inflammation by reducing inflammatory exudation and inhibiting proliferation of fibroblasts and formation of granulation tissue. ${ }^{1-4}$ They have been applied either topically as eyes drops, locally by subconjunctival, peribulbar, or retrobulbar injections, or systemically as oral medications or intravenous or intramuscular injections. Robert Machemer, based on clinical observations and pathogenetic considerations, suggested the intravitreal application of cortisone to locally suppress intraocular inflammation and proliferation of cells, especially in patients with aggressive vitreoretinopathy. ${ }^{5}$ Since cortisone is washed out of the eye within approximately 24 hours after a single intravitreal injection, ${ }^{6}$ Machemer suggested using the crystalline form of cortisone which, owing to an absorption time of about 2 months, provides intraocularly available cortisone for a considerably longer period than the single injection of soluble cortisone. ${ }^{7}$ Experimental studies performed by Graham and Peyman, ${ }^{8}$ and Machemer and co-workers ${ }^{9-16}$ were the basis for the clinical suggestion of performing intravitreal injections of crystalline cortisone. The purpose of the present clinical study was to report on outcome of patients who received an intravitreal injection of crystalline cortisone in combination with pars plana vitrectomy as treatment for proliferative vitreoretinopathy.

\section{Patients and methods}

The study included all 16 patients who underwent pars plana vitrectomy for treatment of proliferative vitreoretinopathy, who received an intravitreal injection of crystalline triamcinolone acetonide at the end of surgery, and who were operated on by the same surgeon (JBJ). There were no other patients undergoing pars plana vitrectomy for proliferative vitreoretinopathy and who received an intravitreal injection of crystalline cortisone. All patients included in the study showed proliferative vitreoretinopathy of grade $\mathrm{C}$ or grade $\mathrm{D}$. Nine of the $16(9 / 16=56.3 \%)$ patients had previously undergone pars plana vitrectomy for proliferative vitreoretinopathy, five of the nine patients had undergone more than one pars plana vitrectomy before being included in the present study. In eight of the nine patients, the previous pars plana vitrectomy had been combined with silicone oil endotamponade. In three patients $(3 / 16,18.8 \%)$, scleral buckling procedures had previously been performed. For four patients $(4 / 16,25 \%)$ presenting with marked proliferative vitreoretinopathy (grade D), the pars plana vitrectomy with intravitreal cortisone injection was the first retinal or vitreoretinal intervention. Pars plana vitrectomy was carried out in general anaesthesia in nine patients. The remaining seven patients underwent surgery with local anaesthesia using the 
Table 1 Composition and clinical data (SD) of the study group undergoing pars plana vitrectomy and intravitreal injection of crystalline cortisone, and the control group

\begin{tabular}{lll}
\hline & Study group & Control group \\
\hline Number & 16 & 144 \\
Age (years) & $51.3(23.8)$ & $50.8(20.3)$ \\
Females/males & $10 / 6$ & $47 / 97$ \\
Right eye/left eye & $10 / 6$ & $80 / 64$ \\
Refractive error (D) & $0.38(1.50)$ & $-0.76(4.30)$ \\
Median; range & $0.00 ; 0.00$ to +6.0 & $0.00 ;-25.0$ to +8.0 \\
Preoperative visual acuity & $0.05(0.07)$ & $0.11(0.18)$ \\
Median; range & $0.02 ; \mathrm{LP}$ to 0.25 & $0.03 ; \mathrm{LP}$ to 1.0 \\
Preoperative IOP (mm Hg) & $16.4(8.5)$ & $12.9(4.4)$ \\
\hline
\end{tabular}

retrobulbar catheter technique for prolonged local anaesthesia and postoperative analgesia by repeatable retrobulbar injections of the anaesthetics through the catheter. ${ }^{17}{ }^{18}$ Mean follow up time was 1.64 (SD 2.15) months (median 1.23 months; range $0.20-9.20$ months).

The study group was compared with a control group consisting of 144 patients with proliferative vitreoretinopathy, who underwent pars plana vitrectomy in the period preceding the start of the present study, and who were operated on by the same surgeon (JBJ) using the same technique, the same surgical instruments, and the same operation theatre. The major difference between the study group and the control group was that for all patients in the control group, pars plana vitrectomy had not been performed before inclusion in the present study. Consequently, the preoperative visual acuity in the control group was significantly $(p<0.05)$ better than in the study group to reduce the effect of a learning curve, the surgeon had performed more than 500 retinal and vitreoretinal operations before the patients in the control group and the patients of the study group were operated. The study group and the control group did not vary significantly $(p>0.05)$ in age and refractive error of the patients. Preoperative intraocular pressure was significantly $(\mathrm{p}<0.05)$ lower in the control group than in the study group, since the patients in the control group, in contrast with eight of 16 patients in the study group, had not previously undergone pars plana vitrectomy with silicone oil endotamponade.

The technique of pars plana vitrectomy performed in the study group as well as in the control group was standardised using three pars plana sclerotomies, removing the vitreous basis, peeling the epiretinal membranes, removing the posterior vitreous surface, performing relaxing retinotomies, using intraoperatively perfluorcarbon liquids, performing retinal endolaser coagulation of retinal breaks and of the edge of retinotomies, and performing transscleral retinal cryocoagulation if retinal lesions in the fundus periphery could not be treated by endolaser coagulation. Silicone oil (viscosity: $5000 \mathrm{cSt}$ ) was used in all eyes included in the study. In the patients in the study group, crystalline triamcinolone acetonide was injected into the silicone oil bubble at the end of surgery through the closed sclerotomies into the vitreous cavity in direction to the ora serrata at the 6 o'clock position. After closure of the conjunctiva, gentamicin was injected subconjunctivally. At the end of surgery, the patients were asked to sit up and to keep an upright position for at least 2 hours after surgery to prevent the cortisone crystals from falling onto the macular region.

The crystalline cortisone was prepared by aspirating the whole volume of a $1 \mathrm{~mm}$ bottle containing $40 \mathrm{mg}$ triamcinolone acetonide (Volon A, Bristol-Myers Squibb)) into a tuberculin syringe of $1 \mathrm{ml}$ volume. The syringe was vertically placed on the operating table for at least 15 minutes. The crystals sedimented into the lower fifth of the syringe. By slowly pressing the syringe which was still kept in the vertical position, the upper $0.8 \mathrm{ml}$ of the syringe containing mostly the vehicle were removed with most of the cortisone crystals left behind in the syringe. The tuberculin syringe was refilled with Ringer's solution, vertically placed for 5 minutes, and again the upper $0.8 \mathrm{ml}$ were removed with most of the cortisone crystals left behind in the lower $0.2 \mathrm{ml}$. The procedure was repeated twice. Then the remaining $0.2 \mathrm{ml}$ with the triamcinolone acetonide crystals were injected into the vitreous cavity. These $0.2 \mathrm{ml}$ contained about $20 \mathrm{mg}$ triamcinolone acetonide. Owing to the dilution process, the $0.2 \mathrm{ml}$ contained about $1 / 625$ of the original volume of the vehicle in a concentration of $1 / 125$ of the original concentration, assuming an equal distribution of the vehicle in the whole volume of the syringe. These figures for volume and concentration of the vehicle injected into the eye will be smaller if one assumes that the inferior part of the vertically placed syringe mainly contained the cortisone crystals.

\section{Results}

In the study group compared with the control group, intraocular inflammation estimated by clinical estimation of anterior chamber flare and of cells in the anterior chamber was lower, and the appearance of the fundus upon ophthalmoscopy in the first postoperative week was clearer. Pain in the first two postoperative days was less in the study group than in the control group. Visual acuity at the end of the follow up period was significantly $(p<0.05)$ better in the control group than in the study group (0.04 (0.03), median 0.03; range, light perception to 0.10 ) versus $0.13(0.08)$ (median 0.08 ; range, light perception to 1.25$)$ ). Intraocular pressure measured at the end of the first postoperative week did not vary significantly between the groups (13.3 (4.6) $\mathrm{mm} \mathrm{Hg}$ (median $12.0 \mathrm{~mm} \mathrm{Hg}$; range 7-22 $\mathrm{mm} \mathrm{Hg}$ ) versus 15.9 (6.3) $\mathrm{mm} \mathrm{Hg}$ (median, $15.0 \mathrm{~mm}$ $\mathrm{Hg}$; range $5-44 \mathrm{~mm} \mathrm{Hg})$ ).

In one patient in the study group $(1 / 16$, $6.3 \%)$, white crystals appeared in the inferior anterior chamber angle resembling a hypopyon. In contrast with patients with a typical postoperative infectious hypopyon, this patient was pain free, there was no marked intraocular inflammation in the upper part of the anterior chamber, and intraocular pressure was in the normal range. In view of the immunosuppressive effect of cortisone, a anterior chamber lavage was performed. Microbiological examination of the aspirate material was unremarkable. In two $(2 / 16,12.5 \%)$ patients, cortisone crys- 
tals had settled on the macular region. Two months after surgery, they had totally resolved. Upon ophthalmoscopy, no tissue damage was detected. With the silicone oil endotamponade in place, a retinal re-detachment was detected in three $(3 / 16,18.8 \%)$ patients at the end of the follow up period.

\section{Discussion}

One of the most important problems still unsolved in clinical ophthalmology is the unwanted proliferation of intraocular and epibulbar tissue such as retinal pigment epithelium cells in the case of proliferative vitreoretinopathy; vascular cells in eyes with ischaemic retinopathies such as proliferative diabetic retinopathy or proliferative retinopathy occurring after central retinal vein occlusion; lens epithelium cells in eyes developing secondary cataract; and fibroblastic cells in patients after antiglaucomatous filtering surgery. This unwanted proliferation of intraocular and episcleral cells is often accompanied and stimulated by intraocular inflammation. Corticosteroids are known to reduce intraocular inflammation and, depending on their concentration, to suppress proliferation of cells. ${ }^{19-22}$ Consequently, they have been used in many ocular conditions given either locally or systemically. To achieve the highest concentration of a drug at its site of action, however, it is best to give it directly into the region of required action. It is, therefore, logical to try to find a method to bring the corticosteroids directly into contact with the tissues on which they should act intraocularly. Thus, this ensures highest possible concentrations and fewer side effects for the rest of the body.

Machemer, Graham, and Peyman, and other researchers have studied the possibility of injecting cortisone directly into the eye, in experimental settings in animals as well as in selected clinical situations in patients. ${ }^{5-162324}$ Additionally, observations of the clinical outcome after accidental intraocular injection of cortisone have been published..$^{25-29}$ In experimental investigations, Machemer and coworkers found that the vehicle, and not the crystalline cortisone itself, can be toxic to the intraocular tissue, ${ }^{12}{ }^{13}$ and they suggested injecting the smallest possible amount of the vehicle. Besides reports on toxicity of the vehicle itself, no study has so far found a direct toxicity of higher concentrations of cortisone injected directly into the eye.

The results of the present study support the findings of the previous investigations. The patients who received the intravitreal injection of crystalline cortisone did not have more but less postoperative intraocular inflammation and pain. This suggests that the intravitreal injection of crystalline cortisone with most of the vehicle removed may not be toxic to the intraocular structures, and that by the intraocular application of a slowly resorbable cortisone, the operation associated intraocular inflammation may be reduced. The finding that postoperative visual acuity was better in the control group than in the study group may be explained by the fact that both groups were not matched for the number of previous vitreoretinal surgeries and preoperative visual acuity. None of the patients in the control group, in contrast with more than $50 \%$ of the $16(9 / 16,56.3 \%)$ patients in the study group, had undergone pars plana vitrectomy before being included in the study. Consequently, preoperative visual acuity was better in the control group than in the study group. Despite the intraocular pressure increasing the effect cortisone can have in some individuals, postoperative intraocular pressure measurements did not differ significantly between the groups. All of these patients had previously received topical steroid treatment and had not reacted with an elevation of intraocular pressure. It suggests that an IOP increasing effect of intravitreal crystalline cortisone may not be a major reason of concern if a cortisone responsiveness has been excluded in the preoperative period.

There are complications of the technique. One pain free patient showed in the early postoperative period a hypopyon-like condition with a whitish semifluid substance accumulating in the inferior part of the anterior chamber angle. In view of the locally immunosuppressive effect of cortisone, a postoperative infectious endophthalmitis was suspected, and an anterior chamber lavage was carried out. The microbiological examination, however, was unremarkable. There are limitations to the present study. The control group was retrospectively formed. The control group and the study group were not matched for preoperative visual acuity and number of previous retinal or vitreoretinal surgeries, with lower vision and more surgeries in the study group than in the control group. It suggests that the patients in the study group showed a more severe grade of proliferative vitreoretinopathy than the patients in the control group. A relative strength of the study is that to reduce the influence of external factors, especially to exclude the effect of a positive learning curve, the study included only patients who were operated by the same surgeon, who had performed more than 500 retinal and vitreoretinal operations before start of the study. Another limitation of the study design is that intraocular inflammation was subjectively assessed by slit lamp biomicroscopy, and that it was not measured using a flare cell meter. All the limitations of the study suggest that the present investigation may only be taken as a pilot study showing the feasibility of the intravitreal injection of crystalline cortisone.

In conclusion, if the results of this pilot study are confirmed by future randomised and prospective investigations, one may infer that the intravitreal injection of crystalline cortisone may not be toxic to the intraocular structures, that it reduces postoperative intraocular inflammation, and that it may be an additional tool in the treatment of proliferative vitreoretinopathy. ${ }^{30}{ }^{31}$ In view of the antiphlogistic, antiproliferative, and intraocular pressure elevating effect of cortisone, possible additional indications of intravitreal injection of crystalline cortisone may include other ocular disorders such as proliferative diabetic 
retinopathy with tractional retinal detachment, long standing therapy resistant uveitis, intraocular surgeries in pseudophakic or aphakic eyes, antiglaucomatous filtering surgery, and pre-phthisical ocular hypotony.

Proprietary interests: none

1 Gordon DM, McLean JM. Effects of pituitary adrenocorticotropin hormone (ACTH) therapy in ophthalmic conditions. $\mathcal{F} A M A$ 1950;142:1271-6.

2 Olson JA, Steffensen EN, Margulis RR, et al. Effect of ACTH on certain inflammatory diseases of the eye. $\mathcal{F A M A}$ 1950;142:1276-8.

3 Woods AC. Clinical and experimental observations on the use of ACTH and cortisone in ocular inflammatory disease. Am f Ophthalmol 1950;33:1325-49.

4 Ruhmann AG, Berliner DL. Influence of steroids on fibroblasts. II. The fibroblast as an assay system for topical fibroblasts. II. The fibroblast as an assay system for topical antiinflammatory potency

5 Machemer R, Sugita G, Tano Y. Treatment of intraocular proliferations with intravitreal steroids. Trans Am Ophthalmol Soc 1979; 77:171-80.

6 Schindler RH, Chandler DB, Thresher R, et al. The clearance of intravitreal triamcinolone acetonide. $A m \mathcal{F}$ Ophthalmol 1982;93:415-17.

7 Machemer R. Five cases in which a depot steroid (hydrocortisone acetate and methylprednisolone acetate) was injected into the eye, Retina 1996;16:166-7.

8 Graham RO, Peyman GA. Intravitreal injection of dexamethasone. Treatment of experimentally induced endophthalmitis. Arch Ophthalmol 1974;92:149-54.

9 Tano Y, Sugita G, Abrams G, et al. Inhibition of intraocular proliferation with intravitreal corticosteroid. Am F Ophthalproliferation with intr.

10 Tano Y, Chandler D, Machemer R. Treatment of intraocular proliferation with intravitreal injection of tramcinolone lar proliferation with intravitreal injection of

11 Tano Y, Chandler DB, McCuen BW, et al. Glucocorticosteroid inhibition of intraocular proliferation after injury. $A m \mathcal{F}$ Ophthalmol 1981;91:184-9.

12 McCuen BW 2d, Bessler M, Tano Y, et al. The lack of toxicity of intravitreally administered triamcinolone acetonide. Am $\mathcal{F}$ Ophthalmol 1981;91:785-8.

13 Hida T, Chandler D, Arena JE, et al. Experimental and clinical observations of the intraocular toxicity of commercial corticosteroid preparations. Am f Ophthalmol 1986, 101:190-5.

14 Chandler DB, Hida $\mathrm{T}$, Sheta $\mathrm{S}$, et al. Improvement in efficacy of corticosteroid therapy in an animal model of proliferative vitreoretinopathy by pretreatment. Graefes Arch Clin Exp Ophthalmol 1987;225:259-65.
15 Coats ML, Peyman GA. Intravitreal corticosteroids in the treatment of exogenous fungal endophthalmitis. Retina 1992;12:46-51.

16 Antoszyk AN, Gottlieb JL, Machemer R, et al. The effects of intravitreal triamcinolone acetonide on experimental preretinal neovascularization. Graefes Arch Clin Exp Ophthalmol 1993;231:34-40.

17 Jonas JB, Hemmerling TM, Budde WM, et al. Postoperative analgesia by re-injections of local anesthetic through an indwelling retrobulbar catheter. Am f Ophthalmol 2000; 129:54-8.

18 Jonas JB, Budde WM, Dinkel M, et al. Indwelling temporary retrobulbar catheter for long-lasting titrable local anesthesia. Arch Ophthalmol 2000;(in press).

19 Pratt WB, Arnow L. The effect of glucocorticoids on protein and nucleic acid synthesis in mouse fibroblasts growing in vitro. F Biol Chem 1966;241:5244-50.

20 Cornman I. Selective damage to fibroblasts by desoxycorticosterone in cultures of mixed tissues. Science 1951;113: 37-9.

21 Hefferman JT, Futterman S, Kalina RE. Dexamethasone inhibition of experimental endothelial cell proliferation in retinal venules. Invest Ophthalmol Vis Sci 1978;17:565-8.

22 Gasset AR, Lorenzetti WC, Ellison EM, et al. Quantitative corticosteroid effect on corneal wound healing. Arch Ophthalmol 1969;81:589-91.

23 Machemer R. Proliferative vitreoretinopathy (PVR): a personal account of its pathogenesis and treatment. Proctor lecture. Invest Ophthalmol Vis Sci 1988;29:1771-83.

24 Binder S. Klinische und experimentelle Ergebnisse in der Behandlung der massiven periretinalen Proliferation (MPP), der prognostisch ungünstigsten Form der Netzhaütösung. [Clinical and experimental results in the treatment of massive periretinal proliferation (MPP), a retinal detachment with a most unfavorable?. Wien Klin Wochenschr (Suppl) 1982;139:1-32.

25 Giles CL. Bulbar perforation during periocular injection of corticosteroids. Am f Ophthalmol 1974;77:438-41.

26 McLean EB. Inadvertent injection of corticosteroid into the choroidal vasculature. Am f Ophthalmol 1975;80:835-7.

27 Zinn KM. Iatrogenic intraocular injection of depot corticosteroids and its surgical removal using the pars plana approach. Ophthalmology 1981;88:13-7.

28 Ghopal L, Bhende M, Sharma T. Vitrectomy for accidental intraocular steroid injection. Retina 1995;15:295-9.

29 Zwaan J. The long-term effects of an accidental injection of depot corticosteroids in an infant eye. Ophthalmic Surg Lasers 1997;28:1017-9.

30 Blumenkranz MS, Ophir A, Claffin AJ, et al. Fluorouracil for the treatment of massive periretinal proliferation. $A m \mathcal{F}$ Ophthalmol 1982;94:458-67.

31 Wiedemann P, Kirmani M, Santana M, et al. Control of experimental massive periretinal proliferation by daunomycin: does-response relation. Graefes Arch Clin Exp Ophthalmol 1983;220:233-5. 